\title{
LIPSCHITZ STRATIFICATION OF REAL ANALYTIC SETS
}

\author{
ADAM PARUSIŃSKI \\ Institute of Mathematics, Gdanisk University, \\ Gdanisk, Poland
}

In this paper we shall prove the existence of a Lipschitz stratification for germs of real analytic sets. The concept of Lipschitz stratification was introduced in [1] by Mostowski, where he proved the existence in a complex case. Lipschitz stratifications are interesting, because they ensure a Lipschitz equisingularity along every stratum.

Our proof will be based on the result of Mostowski and a crucial lemma which estimates the distance to a complex analytic set by the distance to a real one.

Often we shall not distinguish between a set and its germ at the origin. The letter $C$ will denote different constants.

\section{Lipschitz stratifications}

In this section we repeat without proofs the results of Mostowski [1]. By a stratification $\mathscr{S}$ of $\left(\boldsymbol{C}^{n}, 0\right)\left(\left(\boldsymbol{R}^{n}, 0\right)\right.$ respectively) we shall mean a family of germs of analytic sets $X^{j} \subset C^{n}$ (where $j=\operatorname{dim} X^{j}$ ) such that $X^{j} \subset X^{j+1}$ and $\dot{X}^{j}=X^{j} \backslash X^{j-1}$ is smooth. Let $\varrho_{j}(q)$ denote $\operatorname{dist}\left(q, X^{j}\right)$.

Definition 1. A chain for a point $q \in \dot{X}^{j}$ is a decreasing sequence of indices $j_{s}$ and points $q_{j_{s}} \in \dot{X}^{j_{s}}$ such that $j_{1}=j, q_{j_{1}}=q$ and $j_{s}$ is the greatest number for which

$$
\begin{gathered}
\varrho_{k}(q) \geqslant 2 \varrho_{j_{s}}(q) \quad \text { for all } k<j_{s}, \\
\left|q-q_{j_{s}}\right|=\varrho_{j_{s}}(q) .
\end{gathered}
$$

It is easy to check for a chain the following inequalities:

$$
\frac{1}{2} \varrho_{k}(q) \leqslant \varrho_{k}\left(q_{j_{s}}\right) \leqslant \frac{3}{2} \varrho_{k}(q) \quad \text { for } k<j_{s} \text { and all } s \text {. }
$$



$=I-P_{q}$.

For $q \in \dot{X}^{j}$ let $P_{q}$ denote the orthogonal projection of $C^{n}$ onto $T_{q} \dot{X}^{j}, P_{q}^{\perp}$

Definition 2. A stratification $\mathscr{S}$ will be called a Lipschitz stratification if there exists a constant $C>0$ such that for every $q \in \dot{X}^{j}$, every chain $q_{j_{1}}$ $=q, q_{j_{2}}, \ldots$ for $q$ and every $q^{\prime} \in \dot{X}^{j}$ such that $\left|q-q^{\prime}\right| \leqslant \frac{1}{2} \varrho_{j-1}(q)$ the following estimates hold for every $k$ :

$$
\begin{aligned}
& \left|P_{q_{j_{1}}}^{\perp} P_{q_{j_{2}}} \ldots P_{q_{j_{k}}}\right| \leqslant C\left|q_{j_{1}}-q_{j_{2}}\right| / \varrho_{j_{k}-1}\left(q_{j_{1}}\right), \\
& \left|\left(P_{q}-P_{q^{\prime}}\right) P_{q_{j_{2}}} \ldots P_{q_{j_{k}}}\right| \leqslant C\left|q-q^{\prime}\right| / \varrho_{j_{k}-1}(q) .
\end{aligned}
$$

DEFINITION 3. We call a stratification $\mathscr{S}$ compatible with an analytic set $X$ is a union of components of $\dot{X}^{\circ} \mathbf{s}$.

THEOREM 1 (Mostowski [1]). For every finite family of germs of analytic subsets of $C^{n}$ there is a Lipschitz stratification compatible with each of them.

Theorem 1 has two interesting corollaries:

Corollary 1. Let $\mathscr{P}$ be a Lipschitz stratification and $v$ a Lipschitz vector field on $X^{j}$. Then there exists a Lipschit $z$ vector field $w$ on $C^{n}$ such that $w \mid X^{j}=v$ and for every $k$ and $q \in \dot{X}^{k}, w(q) \in T_{q} \dot{X}^{k}$.

Corollary 2. Let $X \subset C^{n} \times C^{m}$ be a germ of an analytic set at the origin and $p: C^{n} \times C^{m} \rightarrow C^{m}$ be the standard projection. There is a proper analytic subset $T$ of $C^{m}$ such that $X$ is Lipschitz equisingular outside $T$ (i.e., for every $t \in C^{m} \backslash T$ there exists an open neighbourhood $U$ of $t$ such that

$$
\left(p^{-1}(U), p^{-1}(U) \cap X,\{0\} \times U\right) \stackrel{\text { Lip }}{\approx}\left(p^{-1}(t) \times U,\left(p^{-1}(t) \cap X\right) \times U,\{0\} \times U\right)
$$

and this Lipschitz homeomorphism is compatible with the standard projections on $U)$.

If we examine the proof of Theorem 1 more carefully we can obtain the following result:

Remark 1. Theorem 1 still holds if we require (L1k) for $q_{j_{1}}$ arbitrary and $\left\{q_{j_{2}}, \ldots, q_{j_{k}}\right\}$ a chain and (L2k) for $q^{\prime}$ arbitrary and $\left\{q_{j_{1}}, \ldots, q_{j_{k}}\right\}$ a chain. Moreover, we can change the conditions in the definition of a chain as follows:

(i) $j_{2}$ is the greatest number for which

$$
\varrho_{k}(q) \geqslant 2 \varrho_{J_{2}}(q) \quad \text { for all } k<j_{2}
$$

(ii) $\left|q-q_{j_{2}}\right|=\varrho_{j_{2}}(q)$;

(iii) $\left\{q_{j_{2}}, \ldots, q_{j_{k}}\right\}$ is a chain (inductive definition), so each part of a chain is also a chain. For such chains we have also estimates similar to (3). 
Remark 2. For a chain $\left\{q_{j_{2}}, \ldots, q_{j_{k}}\right\}$ and $q_{j_{1}}$ arbitrary $\left(j_{1}>j_{2}\right)$ in (L1k) we obtain equivalent inequalities for the following right sides (constants may be different):
(A) $C\left|q_{j_{1}}-q_{j_{2}}\right| / \varrho_{j_{k}-1}\left(q_{j_{1}}\right)$
(B) $C\left|q_{j_{1}}-q_{j_{2}}\right| / \varrho_{j_{k}-1}\left(q_{j_{2}}\right)$;
(C) $C\left|q_{j_{1}}-q_{j_{2}}\right| / \operatorname{dist}\left(\left\{q_{j_{1}}, q_{j_{2}}\right\}, X^{j_{k}-1}\right)$;
(D) $C\left|q_{j_{1}}-q_{j_{2}}\right| / \varrho_{j_{k}-1}\left(q_{j_{k}}\right)$.

A similar result holds for (L2k).

Proof. We prove the equivalence for $(\mathrm{A})$ and $(\mathrm{C})$. It is obvious that if the inequality holds for (A) then it holds for (C). We assume that the inequality holds for $(\mathrm{C})$. We consider two cases:

Case 1. $4\left|q_{j_{1}}-q_{j_{2}}\right| \geqslant \varrho_{j_{k}-1}\left(q_{j_{1}}\right)$, then the inequality holds for (A) trivially, because the left side of (L1k) is bounded by 1 .

Case 2. $\quad 4\left|q_{j_{1}}-q_{j_{2}}\right| \leqslant \varrho_{j_{k}-1}\left(q_{j_{1}}\right)$, then

$$
\left(\operatorname{dist}\left(\left\{q_{j_{1}}, q_{j_{2}}\right\}, X^{j_{k}-1}\right)\right)^{-1} \leqslant 2\left(\varrho_{j_{k}-1}\left(q_{j_{1}}\right)\right)^{-1 / 2},
$$

thus the inequality holds for (A).

The rest of the proof is left to the reader.

We call a complex analytic subset of $\boldsymbol{C}^{n}$ "real" if it is a complexification of a real analytic subset of $\boldsymbol{R}^{n}$.

Remark 3. If a family of sets from the statement of Theorem 1 is "real" then a Lipschitz stratification can be chosen "real".

Remark 4. In [1] Mostowski approximates dist $\left(\cdot, X^{j}\right)$ by some semianalytic function. This is important for the proof but not for the result.

\section{Estimates of distance}

We want to prove the existence of a Lipschitz stratification for real analytic sets by complexification. First we must see how the sides of (L1k), (L2k) change after complexification.

If $Y \subset \boldsymbol{R}^{n}$ is an analytic subset of $\boldsymbol{R}^{n}$ then we denote by $\tilde{Y}$ its complexification. In general, the sign $\tilde{\mathbf{x}}$ will denote passing from the real to the complex case. For example, if $q \in Y \backslash$ Sing $Y$ then $\tilde{P}_{q}$ will denote the orthogonal projection of $C^{n}$ onto $T_{q} \tilde{Y}$ and $P_{q}$ the orthogonal projection of $R^{n}$ onto $T_{q} Y$.

It is easy to see that if $\mathscr{S}=\left\{X^{j}\right\}_{0 \leqslant j \leqslant n}$ is a stratification of $\left(\boldsymbol{R}^{n}, 0\right),\left\{q_{j_{s}}\right\}$ some sequence of points $\left(q_{j_{s}} \in \dot{X}^{j_{s}}\right)$ and $\tilde{\mathscr{T}}=\left\{\tilde{X}^{j}\right\}_{0 \leqslant j \leqslant n}$ a stratification of 
$\left(C^{n}, 0\right)$ formed by the complexifications of $X^{j}$ then

$$
\left|\tilde{P}_{q_{j_{1}}}^{\perp} \tilde{P}_{q_{j_{2}}} \ldots \tilde{P}_{q_{j_{k}}}\right|=\left|P_{q_{j_{1}}}^{\perp} P_{q_{j_{2}}} \ldots P_{q_{j_{k}}}\right|
$$

and a similar equality holds for the left side of $(\mathrm{L} 2 \mathrm{k})$.

So if we want to construct a Lipschitz stratification in real case we must estimate $\operatorname{dist}(q, \tilde{X})\left(q \in \boldsymbol{R}^{n}, X \subset \boldsymbol{C}^{n}\right)$ by a distance to some real analytic set.

THEOREM 2. Let $\tilde{X}$ be a germ of a k-dimensional analytic subset of $C^{n}$ and $X=\tilde{X} \cap R^{n}$. Let $\tilde{Y}$ be a germ of a complex analytic subset of $\tilde{X}$ and $\operatorname{dim}_{c} \tilde{Y} \leqslant k-1$. Then there exists a germ $Y$ of an analytic subset of $X$ and $a$ constant $C$ such that

(i) $\operatorname{dim}_{R} Y \leqslant k-1$;

(ii) $\operatorname{dist}(q, Y) \leqslant C \operatorname{dist}(q, \tilde{Y})$ for $q \in X$.

THEOREM 3. Let $\tilde{Y}$ be a germ of a complex analytic subset of $\boldsymbol{C}^{n}$. Then there is $Y$ a germ of an analytic subset of $R^{n}$ such that

(i) $\operatorname{dim}_{\boldsymbol{R}} Y \leqslant \operatorname{dim}_{c} \tilde{Y}$

(ii) $\operatorname{dist}(q, Y) \leqslant C \operatorname{dist}(q, \bar{Y})$ for $q \in \boldsymbol{R}^{n}$.

The distance from a real point to a complex analytic set is illustrated in Figure 1. The situation of Figure 2 cannot happen.

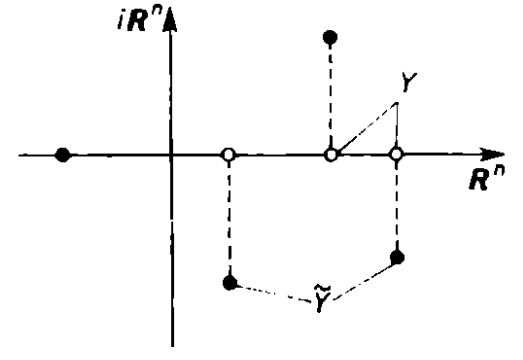

Fig. 1

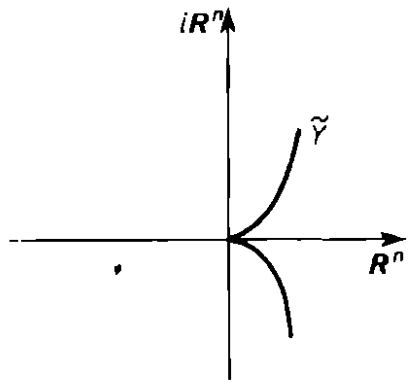

Fig. 2

The proof of Theorem 2 is based on the concept of regular projections introduced by Mostowski ([1]).

\section{Regular projections}

Definition 4. Let $X \subset C^{n}$ be a hypersurface with reduced equation $F$ $=0$. A projection $\pi=\pi_{\xi}: C^{n} \rightarrow C^{n-1}$ parallel to a vector $(\xi, 1)\left(\xi \in C^{n-1}\right)$ will be called $(C, \varepsilon)$-regular with respect to $X$ at $q \in C^{n}$ if

(i) $\pi \mid X$ is finite;

(ii) there exist analytic functions $\lambda_{j}(\eta)$ defined for $|\eta-\xi|<\varepsilon$ such that $F\left(q+\lambda_{j}(\eta)(\eta, 1)\right)=0$, every solution of $F(q+\lambda(\eta, 1))=0$ is of the form $\lambda$ $=\lambda_{j}(\eta)$ and $\lambda_{j}(\eta) \neq \lambda_{k}(\eta)$ for all $\eta$ and $j \neq k,\left|D \lambda_{j}\right| \leqslant C\left|\lambda_{j}\right|$. 
Similarlly $\pi$ will be called regular with respect to $X$ along a curve $q_{t}$ if for some $C, \varepsilon$ it is $(C, \varepsilon)$ regular with respect to $X$ at every point of $q_{t}(t$ small, $t \neq 0$ ).

Choose the $x_{n}$-axis so that $F$ doesn't vanish on it. Let the germ at 0 $(x, \xi, \lambda) \leadsto F(x+\lambda(\xi, 1))$ be equivalent to a distinguished polynomial $W$ with respect to $\lambda$. Let $W$ be defined for $x \in K(a)=\left\{x \in C^{n} ;|x|<a\right\}, \xi \in B(a)$ $=\left\{\eta \in C^{n-1} ;|\eta|<a\right\}$. The following proposition was proved in Mostowski [1].

Proposition 1. There is a finite subset of $B(a), \xi_{1}, \ldots, \xi_{N}$, such that for every complex analytic curve $q_{1}$ in a small neighbourhood of 0 there is a $j$ such that $\pi_{\xi_{j}}$ is regular along $q_{t}$. Furthermore, we can choose $\xi_{1}, \ldots, \xi_{N}$ real.

Let $X \subset C^{n}, B(a)$ be as above, $\xi \in B(a)$. Let $\operatorname{dist}_{\xi}(x, X)$ denote $\operatorname{dist}\left(x, X \cap \pi_{\xi}^{-1}\left(\pi_{\xi}(x)\right)\right)$ (the distance to $X$ in $(\xi, 1)$ direction).

Proposition 2. Let $X, \xi_{1}, \ldots, \xi_{N}$ be as in Proposition 1. Then there exists a constant $C>0$ such that

$$
\underset{j}{\min }\left\{\operatorname{dist}_{\xi_{j}}(x, X)\right\} \leqslant C \operatorname{dist}(x, X) \quad \text { for } x \text { close to } 0 .
$$

Proof. Suppose the proposition were false. Then by the curve selection lemma (see kojasiewicz [3]) we could find a real analytic curve $q_{\mathrm{t}}$ such that:

$$
\underset{j}{\min }\left\{\operatorname{dist}_{\xi_{j}}(q(t), X)\right\} / \operatorname{dist}(q(t), X) \rightarrow \infty, \quad t \rightarrow 0
$$

and $q(t) \in X$ for $t \neq 0$. (We must approximate the distance function by some semi-analytic function $\varphi$ satisfying

$$
\frac{1}{2} \operatorname{dist}(x, X) \leqslant \varphi(x) \leqslant 2 \operatorname{dist}(x, X) .
$$

The existence of a such function is clear from Lojasiewicz [3]).

We can extend this curve to a complex analytic curve which we also denote by $q_{t}$. By Proposition 1 we can choose $\xi_{j}$ such that $\pi_{\xi_{j}}$ is regular along $q_{t}$. Let us assume for simplicity that $\pi=\pi_{\xi_{j}}: C^{n} \rightarrow C^{n-1}$ is the standard projection. Let $C_{q}$ be a cone $\{q+\lambda(\xi, 1) ; \lambda \in C,|\xi|<\varepsilon / 2\}$ and $q=q_{t}$ for some $t=0$. Then it is easy to see from the implicit function theorem that $C_{q} \cap X$ is the disjoint sum of the graphs of analytic functions $\varphi_{j}: K\left(\pi(q),\left|\lambda_{j}(0)\right| \delta\right) \rightarrow C$ (where the constant $\delta>0$ depends only on $C, \varepsilon, n$ ) (see for details Mostowski [1]). Hence $\pi^{-1}\left(K\left(\pi(q), \min _{i}\left|\lambda_{i}(0)\right| \delta\right)\right) \backslash C_{q}$ cannot contain any point of $X$, so $\operatorname{dist}_{\xi_{j}}\left(q_{t}, X\right) \leqslant C \operatorname{dist}\left(q_{t}, \underset{X}{X}\right)$ for some $C>0$ and every $t \neq 0$, but this contradicts (5). 


\section{Proof of Theorem 2}

We first prove the theorem for $X=R^{n}\left(\tilde{X}=C^{n}\right)$. We can assume that $\tilde{Y}$ is a hypersurface in $\boldsymbol{C}^{n}$. Let the vectors $\xi_{1}, \ldots, \xi_{N} \in \boldsymbol{R}^{n-1}$ be chosen for $\tilde{Y}$ as in the statement of Proposition 2. Let $\pi=\pi_{\xi_{j}}$ and assume for simplicity that $\pi$ is the standard projection.

For the product $C^{n}=C^{n-1} \times C$ let $\tilde{Y}_{j}=\left(R^{n-1} \times C\right) \cap \tilde{Y}$ and let $p_{j}: R^{n-1}$ $\times \boldsymbol{C} \rightarrow \boldsymbol{R}^{n-1} \times \boldsymbol{R}$ be the standard projection $(y, z) \rightsquigarrow(y, \operatorname{Re}(z)) . p_{j}\left(\tilde{Y}_{j}\right)$ is a semi-analytic set (see Łojasiewicz [3]) and let $Y_{j}$ be the smallest analytic set containing $p_{j}\left(\tilde{Y}_{j}\right)$ (then $\operatorname{dim} Y_{j} \leqslant k-1$ ).

Let us define $Y=\bigcup_{j} Y_{j}$. We see that for $x \in \boldsymbol{R}^{n}$

$$
\begin{aligned}
\operatorname{dist}(x, X) & =\min _{j} \operatorname{dist}\left(x, Y_{j}\right) \stackrel{\text { 监 }}{\underset{j}{\min } \operatorname{dist}_{\xi_{j}}\left(x, \tilde{Y}_{j}\right)} \\
& =\min _{j} \operatorname{dist}_{\xi_{j}}(x, \tilde{Y}) \stackrel{\text { (iii) }}{\lessgtr} C \operatorname{dist}(x, \tilde{Y}),
\end{aligned}
$$

where (i) follows by construction of $Y_{j}$ and (ii) by Proposition 2. This proves the theorem for $X=R^{n}$.

Now we assume that $\operatorname{dim} X=k<n$ (if $\operatorname{dim} X<k$ the theorem is obvious). Let $\tilde{X}=\tilde{X}_{n} \subset \tilde{Z}^{n-1}$, where $\tilde{Z}^{n-1}$ is a hypersurface in $C^{n}$, and let $\xi_{1}^{n-1}, \ldots, \xi_{N_{n-1}^{n-1}}^{n-1}$ be a family of real vectors as in the statement of Proposition 1. We denote by $\tilde{X}_{n-1}$ the set $\bigcup_{j} \pi_{\xi_{j}}\left(\tilde{X}_{n}\right)$ and let $\tilde{Z}^{n-2}$ be a hypersurface in $C^{n-1}$ containing $\tilde{X}_{n-1}$. We can choose for $\tilde{Z}^{n-2}$ vectors $\xi_{1}^{n-2}, \ldots, \xi_{N_{n-2}-2}^{n_{n}}$ and so on. In this way we construct a sequence of sets $\tilde{X}_{n}, \ldots, \tilde{X}_{k+1}, \tilde{X}_{k}=C^{k}$ each of (complex) dimension $k$ and hypersurfaces $\tilde{Z}^{n-1}, \ldots, \tilde{Z}^{k}$ such that $\tilde{X}_{j} \subset \tilde{Z}^{j-1} \subset C^{j}$. In the same way we define $\tilde{Y}_{n-1}, \ldots, \tilde{Y}_{k}\left(\tilde{Y}_{s}=\bigcup_{j} \pi_{\xi_{j}}\left(\tilde{Y}_{s+1}\right)\right)$. Using the special case considered above, we can find an analytic subset $Y_{k}^{\prime}$ of $\boldsymbol{R}^{k}\left(\operatorname{dim} Y_{k}^{\prime} \leqslant k-1\right)$ such that

$$
\operatorname{dist}\left(x, Y_{k}^{\prime}\right) \leqslant C \operatorname{dist}\left(x, \tilde{Y}_{k}\right)
$$

for $x \in R^{k}$ and some $C>0$.

Now we pass to the real domain.

We inductively define $X_{n}=X, X_{n-1}=\bigcup_{j} \pi_{\xi_{j}}\left(X_{n}\right)$, and so on. $X_{j}$ is a $k$-dimensional semi-analytic subset of $\boldsymbol{R}^{j}$. We also define

$$
\begin{gathered}
T_{n-1}=\bigcup_{j}\left\{\text { critical values of } \pi_{\xi_{j}} \mid X_{n}\right\}, \\
T_{n-2}=\bigcup_{j}\left\{\left(\text { critical values of } \pi_{\xi_{j}} \mid X_{n-1}\right) \cup \pi_{\xi_{j}}\left(T_{n-1}\right)\right\},
\end{gathered}
$$

and so on. Then $T=T_{k} \cup \operatorname{Sing}\left(X_{k}\right)$ is a semi-analytic subset of $X_{k}$ and 
$\operatorname{dim} T \leqslant k-1$. Let. $Y_{k}$ be the smallest analytic subset which contains $Y_{k}^{\prime} \cup T$ $\left(\operatorname{dim} Y_{k} \leqslant k-1\right)$, so for $x \in X_{k}$

$$
\operatorname{dist}\left(x, X_{k} \cap Y_{k}\right) \leqslant \operatorname{dist}\left(x, Y_{k}^{\prime}\right) \leqslant C \operatorname{dist}\left(x, \tilde{Y}_{k}\right) \text {. }
$$

We define $Y_{k+1}=\bigcup_{j} \pi_{\xi_{j}}^{-1}\left(Y_{k}\right)$, and so on. We shall prove that for $x \in X$

$$
\operatorname{dist}\left(x, Y_{n} \cap X\right) \leqslant C \operatorname{dist}\left(x, \tilde{Y}_{n}\right) .
$$

Suppose that (8) is false. Then by the curve selection lemma there is a real analytic curve $q$, such that

$$
\operatorname{dist}\left(q_{t}, \tilde{Y}\right) / \operatorname{dist}\left(q_{t}, Y_{n} \cap X\right) \rightarrow 0, \quad t \rightarrow 0,
$$

and $q_{t} \notin Y_{n} \cap X$ for $t \neq 0$. We can extend $q_{t}$ to a complex analytic curve and use Proposition 1. We choose a sequence of regular projections $\pi_{\xi^{n-1}}$ for $q_{t}$, $\pi_{k n-2}$ for $\pi_{\xi n-1}\left(q_{t}\right)$, and so on. We assume, for simplicity of notation, that these projections are standard, $\pi^{j-1}=\pi_{\xi^{j-1}}: C^{j} \rightarrow C^{j-1}$.

We fix $q=q_{t}$ for some small $t \neq 0$. Let us denote $\pi^{j} \circ \ldots \circ \pi^{n-1}(q)$ by $q_{j}$. Let $y_{k}$ be the nearest point of $Y_{k} \cap X_{k}$ to $q_{k}$ and $l$ the interval joining these points. $l \backslash\left\{y_{k}\right\} \subset X_{k} \backslash T_{k}$, so we can lift $l$ to $l_{k+1}$ a curve in $X_{k+1}$ joining $q_{k+1}$ and some point $y_{k+1}$ of $Y_{k+1} \cap X_{k+1}$. From the regularity of $\pi^{k}$ at $q_{k+1}$ we have

$$
\left|y_{k+1}-q_{k+1}\right| \leqslant C\left|y_{k}-q_{k}\right|
$$

(since $y_{k+1}$ is outside a cone $\left.\left\{q_{k+1}+\lambda(\eta, 1) ; \lambda \in C,|\eta| \leqslant \varepsilon / 2\right\}\right)$. Since $l_{k+1} \backslash\left\{y_{k+1}\right\} \subset X_{k+1} \backslash T_{k+1}$ we can continue a lifting and finally obtain

$$
\operatorname{dist}\left(q, Y_{n} \cap X\right) \leqslant C \operatorname{dist}\left(q_{k}, Y_{k} \cap X_{k}\right)
$$

so by (7)

$$
\operatorname{dist}\left(q, Y_{n} \cap X\right) \leqslant C \operatorname{dist}\left(q_{k}, Y_{k} \cap X_{k}\right) \stackrel{(7)}{\leqslant} C^{\prime} \operatorname{dist}\left(q_{k}, \tilde{Y}_{k}\right) \leqslant C^{\prime \prime} \operatorname{dist}(q, \tilde{Y})
$$

for $q=q_{t}$ ( $t \neq 0$ arbitrary) and $C^{\prime \prime}$ doesn't depend on $t$, in contradiction to (9).

\section{Proof of Theorem 3}

The proof is by induction on $n-j\left(j=\operatorname{dim}_{\mathbf{C}} \tilde{Y}\right)$. For $n-j=1$ the theorem follows from Theorem 2 . Let $n-j>1$. We can find an analytic set $\tilde{Z}$ such that $\tilde{Y} \subset \tilde{Z}$ and $\operatorname{dim} \tilde{Z}=\operatorname{dim} \tilde{Y}+1$. Then by the inductive assumption there is an analytic subset $T$ of $\boldsymbol{R}^{n}$ satisfying

$$
\begin{aligned}
\operatorname{dim} T & \leqslant j+1, \\
\operatorname{dist}(q, T) & \leqslant C \operatorname{dist}(q, \tilde{Z}) \quad \text { for } q \in \boldsymbol{R}^{n} .
\end{aligned}
$$


Let $\tilde{T}$ be the complexification of $T$. Using Theorem 2 for $\tilde{X}=\tilde{Z} \cup \tilde{T}$ and $\tilde{Y}$ we find $Y \subset R^{n}$ such that

$$
\begin{aligned}
& \operatorname{dim} Y \leqslant j, \\
& \operatorname{dist}(x, Y) \leqslant \tilde{C} \operatorname{dist}(x, \tilde{Y}) \quad \text { for } x \in T \subset \tilde{X} \cap R^{n} .
\end{aligned}
$$

We shall prove that $Y$ satisfies the statement of the theorem.

Let $q \in \boldsymbol{R}^{n}$, dist $(q, T)=|q-x|$ for some $x \in T$. We consider two cases.

Case 1. $\operatorname{dist}(q, Y) \leqslant 2(1+\tilde{C})|x-q|$ then

$$
\operatorname{dist}(q, Y) \leqslant 2(1+\tilde{C}) \operatorname{dist}(q, T) \leqslant \hat{C} \operatorname{dist}(q, \tilde{Z}) \leqslant \hat{C} \operatorname{dist}(q, \tilde{Y}) .
$$

Case 2. $\operatorname{dist}(q, Y) \geqslant 2(1+\tilde{C})|x-q|$ then

$$
\begin{aligned}
\operatorname{dist}(q, Y) & \leqslant \operatorname{dist}(x, Y)+|x-q| \leqslant \tilde{C} \operatorname{dist}(x, \tilde{Y})+|x-q| \\
& \leqslant \tilde{C} \operatorname{dist}(q, \tilde{Y})+(1+\tilde{C})|x-q| \leqslant \tilde{C} \operatorname{dist}(q, \tilde{Y})+\frac{1}{2} \operatorname{dist}(q, Y),
\end{aligned}
$$

hence $\operatorname{dist}(q, Y) \leqslant 2 \tilde{C} \operatorname{dist}(q, \tilde{Y})$.

\section{Construction of Lipschitz stratifications}

THEOREM 4. For every finite family of germs at the origin of analytic subsets of $\boldsymbol{R}^{n}$ there is a Lipschitz stratification compatible with each of them.

Proof. We shall give the proof only for one germ, the proof in the general case is similar.

Let $X$ be an analytic subset of some open neighbourhood of 0 in $\boldsymbol{R}^{n}$. Let $\operatorname{dim} X=k$. We define a Lipschitz stratification of $X$ inductively. Suppose that a stratification $X^{n}=R^{n}, X^{n-1}, \ldots, X^{k+1}, X^{k}, X^{k-1}, \ldots$ is chosen in such a way that

$$
\text { (L1k), (L2k) hold for every chain }\left\{q_{j_{s}} \in \dot{X}^{j_{s}}\right\}\left(j_{s}>j\right) \text {. }
$$

Let $\tilde{X}^{n}, \ldots, \tilde{X}^{j}$ be the complexifications of $X^{n}, \ldots, X^{j}$. Then by Theorem 1 we can construct a complex Lipschitz stratification $\hat{\mathscr{S}}$ compatible with $\tilde{X}^{n}, \ldots, \tilde{X}^{j}$. By Theorem 3 we can find an analytic subset $X^{j-1}$ of $R^{n}$ such that for $x \in R^{n}$ we have

$$
\operatorname{dist}\left(x, X^{j-1}\right) \leqslant C \operatorname{dist}\left(x, \hat{X}^{j-1}\right)
$$

and $\operatorname{dim} X^{j-1} \leqslant j-1, \hat{X}^{j-1} \in \hat{\mathscr{Y}}$.

Now we must change $X^{n}, \ldots, X^{j}$ a little to obtain $X^{j} \supset X^{j-1}$. We can do it without affecting the property (11) and the compability of the stratification with $X$.

In fact, we can define $Y^{s}=X^{s} \cup X^{j-1}$ for $s \geqslant j-1$. If $\left\{q_{j_{s}} \in \dot{Y}^{j_{s}}\right\}\left(j_{s}>j\right)$ is a chain then it is a chain in the previous stratification and the estimates (L1k), (L2k) still hold. Changing strata of dimension less then $j-1$ we 
preserve compability. This new stratification will be denoted by $\left\{X^{s}, 0 \leqslant s \leqslant n\right.$.

Now, we shall prove that this stratification satisfies (11) with $j-1$ instead of $j$. We prove only ( $\mathrm{L} / \mathrm{k})$ (the proof of (L2k) is similar).

Let $\left\{q_{j_{s}} \in \dot{X}^{j_{s}},\left(j_{s} \geqslant j, s=1,2, \ldots, t, j_{t}=j\right)\right.$ be a chain. After an arbitrary small perturbation we can assume that for each $s, q_{j_{s}} \in \hat{X}^{j_{s}} \backslash \hat{X}^{j_{s}-1}$. Let $\left\{\tilde{q}_{i_{r}}\right\}$ be a complex chain with respect to $\hat{\mathscr{Y}}^{\prime}=\hat{X}^{n}, \hat{X}^{n-1}, \ldots, \hat{X}^{0}$ for $\tilde{q}_{l_{1}}=q_{j_{2}}$ (in the strong sense of Remark 1).

Let $l_{v} \geqslant j>l_{v+1}$. We shall use the following notation:

$$
\begin{gathered}
\hat{P}_{i}= \begin{cases}\tilde{P}_{\bar{l}_{l_{r}}} & \text { if } i=l_{r} \text { for some } r \text { or } i=j_{1}, \\
\text { Id } & \text { otherwise; }\end{cases} \\
\tilde{P}_{i}= \begin{cases}\tilde{P}_{q_{j_{s}}} & \text { if } i=j_{s} \text { for some } s, \\
\text { Id } & \text { otherwise. }\end{cases}
\end{gathered}
$$

Then we have

$$
\begin{aligned}
& \text { 13) } \quad\left|P_{q_{j_{1}}}^{\perp} \boldsymbol{P}_{q_{j_{2}}} \ldots \boldsymbol{P}_{q_{j_{t}}}\right| \stackrel{(4)}{=}\left|\tilde{P}_{q_{j_{1}}}^{\perp} \tilde{P}_{q_{j_{2}}} \ldots \tilde{P}_{q_{j_{t}}}\right|=\left|\tilde{P}_{j_{1}}^{\perp} \tilde{P}_{j_{1}-1} \ldots \tilde{P}_{j}\right| \\
& \leqslant\left|\hat{P}_{j_{1}}^{\perp} \hat{P}_{j_{1}-1} \ldots \hat{P}_{j}\right|+\sum_{j \leqslant w-j_{2}-1} \underbrace{\left|P_{j_{1}} P_{j_{1}-1} \ldots P_{w+1}\right|\left|\left(\hat{P}_{w}-\tilde{P}_{w}\right) \hat{P}_{w-1} \ldots \hat{P}_{j}\right|}_{A_{w}}
\end{aligned}
$$

Now we consider three cases:

Case 1. $w=j_{s}=l_{r}$ (for some $s, r$ ); then by Remark 1,2 and the properties of chains (the letter $C$ denotes different constants):

$$
\begin{aligned}
\left|\left(\hat{P}_{w}-\tilde{P}_{w}\right) \hat{P}_{w-1} \ldots \hat{P}_{j}\right| & =\mid\left(\tilde{P}_{q_{j_{s}}}^{\perp}-\tilde{P}_{\tilde{q}_{l_{r}}}\right) \tilde{P}_{\tilde{q}_{l_{r}+1}} \ldots \tilde{P}_{\tilde{q}_{l_{v}} \mid} \\
& \leqslant \frac{C\left|q_{j_{s}}-\tilde{q}_{l_{l}}\right|}{\operatorname{dist}\left(\tilde{q}_{l_{r}}, \hat{X}^{l_{v}-1}\right)} \leqslant \frac{C\left|q_{j_{s}}-\tilde{q}_{l_{r}}\right|}{\operatorname{dist}\left(\tilde{q}_{l_{1}}, \hat{X}^{j-1}\right)}
\end{aligned}
$$

so

$$
\begin{aligned}
& A_{w} \leqslant \frac{C\left|q_{j_{1}}-q_{j_{2}}\right|\left|q_{j_{s}}-q_{l_{r}}\right|}{\operatorname{dist}\left(q_{j_{2}}, X^{j_{s-1}-1}\right) \operatorname{dist}\left(\tilde{q}_{1_{1}}, \hat{X}^{j-1}\right)} \\
& \stackrel{(12)}{\leqslant} \frac{C\left|q_{j_{1}}-q_{j_{2}}\right|\left|q_{j_{s}}-\tilde{q}_{l_{r}}\right|}{\operatorname{dist}\left(q_{j_{2}}, X^{j_{s}}\right) \operatorname{dist}\left(q_{j_{2}}, X^{j-1}\right)} \stackrel{\text { (*) }}{\lessgtr} \frac{C\left|q_{j_{1}}-q_{j_{2}}\right|}{\operatorname{dist}\left(q_{j_{2}}, X^{j-1}\right)} \text {, }
\end{aligned}
$$

(\#) $\quad\left|q_{j_{s}}-\tilde{q}_{l_{r}}\right| \leqslant C \operatorname{dist}\left(q_{j_{2}}, \hat{X}^{l_{r}}\right)+C \operatorname{dist}\left(q_{j_{2}}, X^{j_{s}}\right) \leqslant C \operatorname{dist}\left(q_{j_{2}}, X^{j_{s}}\right)$.

Case 2. $l_{r-1}>w=j_{s}>l_{r} \geqslant l_{v}$ (for some $\left.s, r\right)$

$$
\begin{aligned}
\left|\left(\hat{P}_{w}-\tilde{P}_{w}\right) \hat{P}_{w-1} \ldots \hat{P}_{j}\right| & =\left|\tilde{P}_{q_{j_{s}}}^{\perp} \tilde{P}_{\tilde{q}_{l_{r}}} \ldots \tilde{P}_{\tilde{q}_{l_{v}}}\right| \\
& \leqslant \frac{C\left|q_{j_{s}}-\tilde{q}_{l_{r}}\right|}{\operatorname{dist}\left(\tilde{q}_{l_{r}}, \hat{X}^{l_{v}-1}\right)} \leqslant \frac{C\left|q_{j_{s}}-\tilde{q}_{l_{r}}\right|}{\operatorname{dist}\left(\tilde{q}_{l_{1}}, \hat{X}^{j-1}\right)}
\end{aligned}
$$


so

$$
A_{w} \leqslant \frac{C\left|q_{j_{1}}-q_{j_{2}}\right|\left|q_{j_{s}}-q_{l_{r}}\right|}{\operatorname{dist}\left(q_{j_{2}}, X^{j_{s-1}-1} \operatorname{dist}\left(\tilde{q}_{l_{1}}, \hat{X}^{j-1}\right)\right.} \leqslant \frac{C\left|q_{j_{1}}-q_{j_{2}}\right|}{\operatorname{dist}\left(q_{j_{2}}, X^{j-1}\right)}
$$

because

$$
\begin{aligned}
\left|q_{j_{s}}-q_{l_{r}}\right| & \leqslant C \operatorname{dist}\left(q_{j_{2}}, X^{j_{s}}\right)+C \operatorname{dist}\left(q_{j_{2}}, \hat{X}^{l_{r}}\right) \\
& \leqslant C \operatorname{dist}\left(q_{j_{2}}, X^{j_{s}}\right)+C \operatorname{dist}\left(q_{j_{2}}, \hat{X}^{j_{s}}\right) \leqslant C \operatorname{dist}\left(q_{j_{2}}, X^{j_{s}-1}\right) .
\end{aligned}
$$

If $w=j_{s}<l_{v}$ then

$$
\begin{aligned}
A_{w} & \leqslant \frac{C\left|q_{j_{1}}-q_{j_{2}}\right|}{\operatorname{dist}\left(q_{j_{2}}, X^{j_{s}-1}-1\right.} \leqslant \frac{C\left|q_{j_{1}}-q_{j_{2}}\right|}{\operatorname{dist}\left(q_{j_{2}}, X^{j_{s}}\right)} \\
& \leqslant \frac{C\left|q_{j_{1}}-q_{j_{2}}\right|}{\operatorname{dist}\left(q_{j_{2}}, \hat{X}^{j_{s}}\right)} \leqslant \frac{C\left|q_{j_{1}}-q_{j_{2}}\right|}{\operatorname{dist}\left(q_{j_{2}}, \hat{X}^{j-1}\right)} \\
& \leqslant \frac{C\left|q_{j_{1}}-q_{j_{2}}\right|}{\operatorname{dist}\left(q_{j_{2}}, X^{j-1}\right)} .
\end{aligned}
$$

Case 3. $j_{s}<w=l_{r}<j_{s-1}$ (for some $s, r$ ). In this case the proof of the estimate

$$
A_{w} \leqslant \frac{C\left|q_{j_{1}}-q_{j_{2}}\right|}{\operatorname{dist}\left(q_{j_{2}}, X^{j-1}\right)}
$$

is similar to the proof of $(15),(17),(18)$ and is left to the reader.

Since

$$
\begin{aligned}
\left|\hat{P}_{j_{1}}^{\perp} \hat{P}_{j_{1}-1} \ldots \hat{P}_{j}\right| & =\left|\tilde{P}_{q_{j_{1}}}^{\perp} \tilde{P}_{\tilde{q}_{l_{1}}} \ldots \tilde{P}_{\tilde{q}_{l_{v}}}\right| \leqslant \frac{C\left|q_{j_{1}}-q_{j_{2}}\right|}{\operatorname{dist}\left(q_{j_{2}}, X^{l_{v}-1}\right)} \\
& \leqslant \frac{C\left|q_{j_{1}}-q_{j_{2}}\right|}{\operatorname{dist}\left(q_{j_{2}}, \hat{X}^{j-1}\right)} \leqslant \frac{C\left|q_{j_{1}}-q_{j_{2}}\right|}{\operatorname{dist}\left(q_{j_{2}}, X^{j-1}\right)}
\end{aligned}
$$

we have (L1k) from (13), (19).

In this way we can construct inductively a stratification which satisfies (11) for $j=-1$, in other words a Lipschitz stratification.

\section{Some remarks}

The same proof works for germs of closed semi-analytic sets, but it fails in the sub-analytic case. So the problem of the existence of a Lipschitz stratification for sub-analytic sets is still open. The second problem is to construct a global Lipschitz stratification, for compact sets for example. It 
seems to be difficult because the Lipschitz stratification constructed above is not "canonical". For example, if $X=\left\{(x, y, z) \in \boldsymbol{R}^{3} ; y^{2}+z^{2}=x^{3}\right\}$ then $X^{2}$ $=X, X^{1}=X^{0}=\{0\}$ is not a Lipschitz stratification. To obtain a Lipschitz stratification we must choose for $X^{1}$ any curve satisfying $C_{0}\left(X^{1}\right) \supset x$-axis

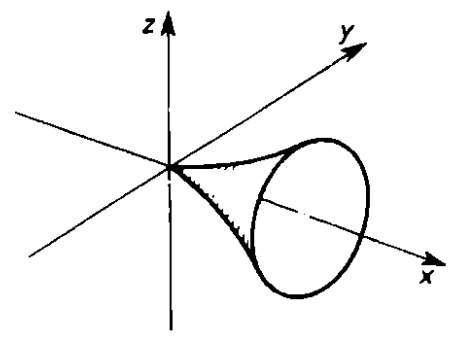

Fig. 3

$\left(C_{0}\left(X^{1}\right)\right.$ denotes the tangent cone to $X^{1}$ at 0 ). (The best general reference to this problem is Mostowski [2]). The methods of Mostowski [1] and the above enable us to construct a global Lipschitz stratification in the algebraic affine case.

I would like to thank Tadeusz Mostowski for calling my interest to the problem and many valuable suggestions.

\section{References}

[1] T. Mostowski, Lipschitz equisingularity, Dissertationes Math. (Rozprawy Mat.) 243 (1985).

[2] -. Tangent cones and Lipschitz stratifications, this volume, 303-322.

[3] S. Lojasiewicz, Ensembles semi-analytiques, Institute des Hautes Études Scientifiques, Bûres-sur-Yvette 1965. 